\title{
FOUR-MANIFOLDS WITHOUT EINSTEIN METRICS
}

\author{
Claude LeBrun
}

\begin{abstract}
It is shown that there are infinitely many compact simply connected smooth 4-manifolds which do not admit Einstein metrics, but nevertheless satisfy the strict Hitchin-Thorpe inequality $2 \chi>3|\tau|$. The examples in question arise as non-minimal complex algebraic surfaces of general type, and the method of proof stems from Seiberg-Witten theory.
\end{abstract}

\section{Introduction}

A smooth Riemannian metric $g$ is said to be Einstein if its Ricci curvature $r$ is a constant multiple of the metric:

$$
r=\lambda g .
$$

Not every 4-manifold admits such metrics. A necessary condition for the existence of an Einstein metric on a compact oriented 4-manifold is that the Hitchin-Thorpe inequality $2 \chi(M) \geq 3|\tau(M)|$ must hold [8, 26, 4]. Moreover, equality can hold only if $M$ manifold is finitely covered by a torus or K3 surface. We will say that $M$ satisfies the strict Hitchin-Thorpe inequality if $2 \chi(M)>3|\tau(M)|$.

The purpose of this note is to prove the following result:

Theorem A. There are infinitely many compact simply connected smooth 4-manifolds which do not admit Einstein metrics, but nevertheless satisfy the strict Hitchin-Thorpe inequality.

The examples we shall consider arise as non-minimal complex surfaces of general type. The proof hinges on scalar curvature estimates that come from Seiberg-Witten theory.

\section{Scalar curvature and topology}

In this section, we will develop a new lower bound for the $L^{2}$-norm of the scalar curvature of Riemannian metrics on a non-minimal complex surfaces of general type. Let us begin by reviewing some definitions and results.

Received November 27, 1995.

Supported in part by NSF grant DMS-9505744. 
Definition 1. Let $M$ be a smooth compact oriented 4-manifold. A polarization of $M$ is a linear subspace $H^{+} \subset H^{2}(M, \mathbb{R})$ on which the restriction of the intersection form is positive-definite, and which is a maximal subspace with this property.

The example of interest is the following: let $g$ be a Riemannian metric, and let $H^{+}(g)$ be the space of harmonic self-dual 2-forms with respect to $g$. Then $H^{+}(g)$ is a polarization. If $H^{+}$is a given polarization, and if $H^{+}(g)=H^{+}$, we will say that $g$ is adapted to $H^{+}$.

If $J$ is an orientation-compatible almost-complex structure on $M, J$ induces a $\operatorname{spin}^{c}$-structure $c$ on $M$, and for every metric $g$ one then has a pair of rank-2 complex vector bundles $V_{ \pm}$which formally satisfy

$$
V_{ \pm}=\mathbb{S}_{ \pm} \otimes L^{1 / 2}
$$

where $\mathbb{S}_{ \pm}$are the left- and right-handed spinor bundles of $g$, and $L$ is the anti-canonical line bundle of $J$. For each unitary connection $\theta$ on $L$, we have a Dirac operator $D_{\theta}: C^{\infty}\left(V_{+}\right) \rightarrow C^{\infty}\left(V_{-}\right)$, and one can then consider the Seiberg-Witten equations [30]

$$
\begin{aligned}
D_{\theta} \Phi & =0 \\
F_{\theta}^{+} & =i \sigma(\Phi)
\end{aligned}
$$

for an unknown section $\Phi$ of $V_{+}$and an unknown unitary connection $\theta$. Suppose that $H^{+}$is a polarization such that the orthogonal projection $c^{+}$of $c_{1}(L)$ into $H^{+}$is non-zero. Let $g$ be any $H^{+}$-adapted metric, and consider the moduli space of solutions of a generic perturbation of the Seiberg-Witten equations modulo gauge equivalence. This moduli space consists of a finite number of oriented points, and the Seiberg-Witten invariant $n_{c}\left(M, H^{+}\right)$is defined to be the number of points in this moduli space, counted with signs. This is independent of all choices. Indeed, if $b^{+}(M)>1$, it is even independent of $H^{+}$.

A Weitzenböck argument yields the following curvature estimate [15]:

Theorem 1. Let $\left(M, H^{+}, c\right)$ be a smooth compact oriented polarized 4manifold with spin ${ }^{c}$ structure such that $n_{c}\left(M, H^{+}\right) \neq 0$. If $c_{1}(L) \in$ $H^{2}(M, \mathbb{R})$ is the anti-canonical class of this spin ${ }^{c}$ structure, let $c_{1}^{+}$be its orthogonal projection to $\mathrm{H}^{+}$with respect to the intersection form. Then every $\mathrm{H}^{+}$-adapted Riemannian metric $g$ satisfies

$$
\int_{M} s^{2} d \mu \geq 32 \pi^{2}\left(c_{1}^{+}\right)^{2}
$$

If $c_{1}^{+} \neq 0$, moreover, equality occurs iff $g$ is Kähler with respect to a ccompatible complex structure and has constant negative scalar curvature. 
Now if $(M, J)$ is a complex surface of Kähler type with $b^{+}>1$, and if $c$ is the $\operatorname{spin}^{c}$ structure induced by $J$, then $n_{c}\left(M, H^{+}\right)=n_{c}(M)=1$. For complex surfaces with $b^{+}=1$, the picture is more complicated, but can be summarized as follows. The set of classes $\alpha \in H^{2}(M, \mathbb{R})$ with $\alpha^{2}:=\alpha \cdot \alpha>0$ consists of two connected components. One component contains the Kähler classes of all Kähler metrics on $M$; let us call the elements of this component future pointing, and the elements of the other past-pointing. Then $n_{c}\left(M, H^{+}\right)=1$ whenever $c_{1}^{+}$is past-pointing; by contrast, if $b_{1}(M)=0$, then $n_{c}\left(M, H^{+}\right)=0$ whenever $c_{1}^{+}$is future pointing $[6,12]$.

We now come to the technical heart of the article:

Theorem 2. Let $X$ be a minimal complex algebraic surface of general type, and let $M=X \# k \overline{\mathbb{C P}}_{2}$ be obtained from $X$ by blowing up $k>0$ points. Then any Riemannian metric on $M$ satisfies

$$
\int_{M} s^{2} d \mu>32 \pi^{2}(2 \chi+3 \tau+k),
$$

where $\chi$ and $\tau$ are respectively the Euler characteristic and signature of $M$.

Proof. Let us think of $M$ concretely as obtained from $X$ by blowing up $k$ distinct points $p_{1}, \ldots, p_{k}$, so that $M$ comes equipped with an integrable complex structure $J$. The key observation [6] is that instead of merely considering this complex structure alone, it is natural to consider $2^{k}$ distinct complex structures, each of which is the pull-back of $J$ via a diffeomorphism $M \rightarrow M$. To this end, choose a biholomorphism between a neighborhood of $p_{j} \in X$ and the unit ball in $\mathbb{C}^{2}=\mathbb{R}^{4}$. Let $\psi_{j}: X \rightarrow X$ be the identity outside this neighborhood, and act by

$$
\left[\begin{array}{cccc}
1 & 0 & 0 & 0 \\
0 & \cos \pi u(\varrho) & 0 & -\sin \pi u(\varrho) \\
0 & 0 & 1 & 0 \\
0 & \sin \pi u(\varrho) & 0 & \cos \pi u(\varrho)
\end{array}\right]
$$

on the ball itself; here $\varrho$ is the distance from the origin in $\mathbb{R}^{4}$, and the smooth function $u$ satisfies $u(\varrho) \equiv 1$ for $\varrho \leq \frac{1}{3}$ and $u(\varrho) \equiv 0$ for $\varrho \geq$ $\frac{2}{3}$. Since $\psi_{j}$ is complex anti-linear in a neighborhood of $p_{j}$, it induces a diffeomorphism $\varphi_{j}: M \rightarrow M$. Assuming that the neighborhoods in question are pairwise disjoint, the $\varphi_{j}$ 's commute with each other, and if $S \subset\{1, \ldots k\}$ is any subset, we may therefore unambiguously define $\varphi_{S}$ to be the composition of those $\varphi_{j}$ 's for which $j \in S$. Now $J_{S}=\varphi_{S}^{*} J$ is an integrable complex structure on $M$ for each $S \subset\{1, \ldots k\}$; for example, $J_{\emptyset}=J$. 
Let $c_{1}(X)$ denote the pull-back to $M$ of the first Chern class of $X$ via the blowing-down map $M \rightarrow X$, and let $E_{1}, \ldots, E_{k}$ be the Poincaré duals of the exceptional divisors corresponding to $p_{1}, \ldots, p_{k}$. The first Chern class of $T_{J_{S}}^{1,0} M$ is then

$$
c_{1}\left(M, J_{S}\right)=c_{1}(X)+\sum \epsilon_{j},
$$

where

$$
\epsilon_{j}=\left\{\begin{array}{cc}
E_{j} & \text { if } j \in S \\
-E_{j} & \text { if } j \notin S
\end{array}\right.
$$

If $g$ is any Riemannian metric on $M$, the projection of $c_{1}\left(M, J_{S}\right)$ into the space $H^{+}(g) \subset H^{2}(M, \mathbb{R})$ of self-dual harmonic 2-forms is therefore $c_{1}\left(M, J_{S}\right)^{+}=c_{1}(X)^{+}+\sum \epsilon_{j}^{+}$. (Note that $c_{1}(X)^{2}=c_{1}^{2}(X)>0$, so that $c_{1}^{+} \neq 0$.) Now choose $S$ so that

$$
c_{1}(X)^{+} \cdot \epsilon_{j}^{+} \geq 0 .
$$

If $c$ is the $\operatorname{spin}^{c}$ structure associated with this choice of $S$, the SeibergWitten invariant of $\left(M, H^{+}, c\right)$ is non-zero [6], and Theorem 1 tells us that

$$
\begin{aligned}
\frac{1}{32 \pi^{2}} \int_{M} s^{2} d \mu & \geq\left(c_{1}\left(M, J_{S}\right)^{+}\right)^{2} \\
& =\left(c_{1}(X)^{+}+\sum \epsilon_{j}^{+}\right)^{2} \\
& =\left(c_{1}(X)^{+}\right)^{2}+2 \sum\left(c_{1}(X)^{+} \cdot \epsilon_{j}^{+}\right)+\left(\sum \epsilon_{j}^{+}\right)^{2} \\
& \geq\left(c_{1}(X)^{+}\right)^{2} \\
& \geq\left(c_{1}(X)\right)^{2}=c_{1}^{2}(X) \\
& =2 \chi+3 \tau+k
\end{aligned}
$$

because the intersection form is positive-definite on $\mathrm{H}^{+}=\mathrm{H}^{+}(g)$.

Now suppose we have a metric $g$ for which this inequality is actually an equality. Then each of the inequalities in the above calculation is an equality, and Theorem 1, applied to the first of these, tells us that $g$ is Kähler with respect to a complex structure $J_{g}$ compatible with $c$, and hence satisfying $c_{1}\left(M, J_{g}\right)=c_{1}\left(M, J_{S}\right)$. By the same reasoning, $\left(\sum \epsilon_{j}^{+}\right)^{2}=0$, and hence $\sum \epsilon_{j}^{+}=0$. In particular, $c_{1}\left(M, J_{S}\right)^{+}=c_{1}\left(M, J_{\tilde{S}}\right)^{+}$, where $\tilde{S}=\{1, \ldots, k\}-S$, so, even if $b^{+}=1$, the Seiberg-Witten invariant of $\left(M, H^{+}, \tilde{c}\right)$ is also non-zero, where $\tilde{c}$ is the $\operatorname{spin}^{c}$ structure determined by $J_{\tilde{S}}$. But since $c_{1}^{+}\left(J_{\tilde{S}}\right)=c_{1}^{+}\left(J_{S}\right)$, Theorem 1 also tells us that $g$ is Kähler with respect to an orientation-compatible complex structure $\tilde{J}_{g}$ such that $c_{1}\left(M, \tilde{J}_{g}\right)=c_{1}\left(M, J_{\tilde{S}}\right)$; and $c_{1}\left(M, J_{\tilde{S}}\right) \neq c_{1}\left(M, J_{S}\right)$, so $J_{g} \neq \tilde{J}_{g}$. Hence $g$ has holonomy in $S U(2)$, and so is hyper-Kähler. But this implies [4] that 
$g$ is Ricci-flat, and so has $s \equiv 0$. But since $c_{1}^{+} \neq 0$, Theorem 1 guarantees that $g$ has negative scalar curvature, and this contradiction shows that our assumption was therefore false; the inequality is always strict.

\section{Einstein metrics}

Theorem 3. Let $X$ be a minimal complex algebraic surface of general type, and let $M=X \# k \overline{\mathbb{C P}}_{2}$ be obtained from $X$ by blowing up $k>0$ points. If $k \geq \frac{2}{3} c_{1}^{2}(X)$, then $M$ does not admit Einstein metrics.

Proof. For any Riemannian metric $g$ on $M$, one has the generalized GaussBonnet formula

$$
2 \chi+3 \tau=\frac{1}{4 \pi^{2}} \int_{M}\left(2\left|W_{+}\right|^{2}+\frac{s^{2}}{24}-\frac{|\stackrel{\circ}{r}|^{2}}{2}\right) d \mu
$$

where $s, \stackrel{\circ}{r}$, and $W_{+}$are respectively the scalar, trace-free Ricci, and selfdual Weyl curvatures of $g$; pointwise norms are calculated with respect to the metric, and $d \mu$ is the metric volume form. If $g$ is an Einstein metric, $\stackrel{\circ}{r}=0$, and Theorem 2 therefore implies that

$$
\begin{aligned}
c_{1}^{2}(X)-k=2 \chi+3 \tau & =\frac{1}{4 \pi^{2}} \int_{M}\left(2\left|W_{+}\right|^{2}+\frac{s^{2}}{24}\right) d \mu \\
& >\frac{32 \pi^{2}}{4 \cdot 24 \pi^{2}}(2 \chi+3 \tau+k) \\
& =\frac{1}{3} c_{1}^{2}(X)
\end{aligned}
$$

so that

$$
\frac{2}{3} c_{1}^{2}(X)>k
$$

contradicting our assumption. Hence $M$ cannot admit an Einstein metric.

Our main result now follows.

Theorem A. Even up to homeomorphism, there are infinitely many smooth simply connected compact 4-manifolds which do not admit Einstein metrics, but nonetheless satisfy the strict Hitchin-Thorpe inequality.

Proof. If $X$ is any minimal complex surface of general type with $c_{1}^{2} \geq 3$, there is then at least one integer $k$ satisfying $c_{1}^{2}>k \geq \frac{2}{3} c_{1}^{2}$. Because of the Miyaoka-Yau inequality $\chi \geq 3 \tau$, the complex surface $M=X \# k \overline{\mathbb{C P}}_{2}$ then automatically satisfies the strict Hitchin-Thorpe inequality $2 \chi>3|\tau|$, but does not admit Einstein metrics by Theorem 3 . 
The oriented-homeomorphism invariant $b^{+}$is invariant under blowing up, so it suffices to produce a sequence of simply connected minimal surfaces $X_{j}$ of general type with $c_{1}^{2} \geq 3$ such that the sequence of integers $b^{+}\left(X_{j}\right)$ is increasing. One such sequence is given by the Fermat surfaces $w^{m}+x^{m}+y^{m}+z^{m}=0$ of degree $m=j+4$, with $b^{+}\left(X_{j}\right)=$ $1+(j+1)(j+2)(j+3) / 3$.

\section{The symplectic case}

In order to keep our discussion as concrete and elementary as possible, we have thus far assumed that our 4-manifolds arose as compact complex surfaces. The proof of Theorem 2, however, only depends on the nonvanishing of certain Seiberg-Witten invariants of $M=X \# k \overline{\mathbb{C} P}_{2}$. Now if $X$ admits a symplectic structure, the symplectic blow-up construction of McDuff [18] supplies a family of such structures on $M$, and a result of Taubes [23] then provides us with the non-vanishing invariants we need to prove the following:

Theorem 4. Let $(X, \omega)$ be a symplectic manifold, and let $M=X \# k \overline{\mathbb{C P}}_{2}$. If $b^{+}(X)=1$, assume that $c_{1}(X) \cdot[\omega]<0$. Then any Riemannian metric on $M$ satisfies

$$
\int_{M} s^{2} d \mu>32 \pi^{2} c_{1}^{2}(X)
$$

Here, of course, $c_{1}(X)$ is the first Chern class of an almost-complex structure adapted to the symplectic structure. The assumption that $c_{1}(X)$. $[\omega]<0$ if $b^{+}=1$ is needed to compensate for the fact that Taubes' proof involves large perturbations of the Seiberg-Witten equations, whereas the relevant scalar curvature estimates stem from the unperturbed equations.

This immediately yields a generalization of Theorem 3 :

Theorem 5. Let $(X, \omega)$ be a symplectic manifold, and let $M=X \# k \overline{\mathbb{C P}}_{2}$. If $b^{+}(X)=1$, assume that $c_{1}(X) \cdot[\omega]<0$. If $k \geq \frac{2}{3} c_{1}^{2}(X)$, then $M$ does not admit an Einstein metric.

Of course, this is a trivial consequence of the Hitchin-Thorpe inequality unless $c_{1}^{2}(X)>0$. On the other hand, it is unnecessarily weak if $X$ is itself the blow-up of another symplectic manifold. In analogy with the EnriquesKodaira classification, it is therefore natural to introduce a definition which characterizes the natural setting for applications of these results:

Definition 2. A minimal symplectic 4-manifold $(X, \omega)$ is of general type if

: (a) $c_{1}^{2}(X)>0$; and

: (b) $c_{1}(X) \cdot[\omega]<0$. 
A symplectic 4-manifold of general type is defined as an iterated symplectic blow-up of such a minimal symplectic manifold of general type.

If $b^{+}>1$, Taubes $[24,25]$ has shown that condition (b) is automatic and that (a) fails only for minimal symplectic manifolds with $c_{1}^{2}=0$. Minimal manifolds of the latter type may be considered to be symplectic generalizations of elliptic surfaces.

\section{Yamabe constants and minimal volumes}

Theorem 1 tells us that any Riemannian metric on a non-minimal surface $M=X \# k \overline{\mathbb{C P}}_{2}$ of general type satisfies the scalar-curvature estimate

$$
\int_{M} s^{2} d \mu>32 \pi^{2} c_{1}^{2}(X)
$$

where $X$ is the minimal model for $M$. In fact, this estimate is sharp. To see this, let us first consider the case in which $X$ does not contain any $(-2)$-curves. This assumption implies [3] that $c_{1}(X)<0$, and hence $[2,31]$ that $X$ admits a Kähler-Einstein metric $\check{g}$; we then have

$$
\int_{X} s_{\check{g}}^{2} d \mu_{\breve{g}}=32 \pi^{2} c_{1}^{2}(X)
$$

Choose disjoint complex coordinate charts centered at the points $p_{1}, \ldots, p_{k}$ which are to be blown up to obtain $M$, with the property that

$$
\check{g}=\delta+O\left(\varrho^{2}\right)
$$

where $\delta$ and $\varrho$ are respectively the Euclidean metric and radius associated with the chart, and set $h_{1}=\delta-\check{g}$. Let $h_{2}$ denote the pull-back of the Fubini-Study metric on $\mathbb{C} P_{1}$ to $\mathbb{C}^{2}-0$ via the tautological projection, and use these same charts to transplant $h_{2}$ to a punctured neighborhood of each of the $p_{1}, \ldots, p_{k}$. Let $\phi: \mathbb{R} \rightarrow \mathbb{R}$ be a non-negative smooth function which is identically 1 on $\left(-\infty, \frac{1}{2}\right)$ and identically 0 on $(1, \infty)$, and, for each sufficiently small $t<0$, let $g_{t}$ be the smooth Riemannian metric on the blow-up $M$ whose restriction to the open dense set $X-\left\{p_{1}, \ldots, p_{k}\right\}$ is given by

$$
g_{t}=\check{g}+\phi\left(\frac{\varrho}{t}\right)\left[h_{1}+t^{4} h_{2}\right] .
$$

For $\varrho<t / 2$, this metric coincides up to scale with the Burns metric [13] on the blow-up of $\mathbb{C}^{2}$ at the origin, and so has scalar curvature $s \equiv 0$; and 
for $\varrho>t$, it coincides with $\check{g}$. In the transition region $\varrho \in(t / 2, t)$, one has

$$
\begin{aligned}
\left\|g_{t}-\delta\right\| & \leq C t^{2} \\
\left\|\mathbb{D} g_{t}\right\| & \leq C t \\
\left\|\mathbb{D}^{2} g_{t}\right\| & \leq C
\end{aligned}
$$

where $\mathbb{D}$ is the Euclidean derivative operator associated with the given coordinate system, and the constant $C$ is independent of $t$. Thus $s^{2}\left(g_{t}\right)$ is uniformly bounded as $t \rightarrow 0$, and since the volume of the annular transition region is of order $t^{4}$, we conclude that

$$
\lim _{t \rightarrow 0^{+}} \int_{M} s_{g_{t}}^{2} d \mu_{g_{t}}=\int_{X} s_{\check{g}}^{2} d \mu_{\check{g}}=32 \pi^{2} c_{1}^{2}(X) .
$$

The bound is therefore sharp, as claimed.

Even if $X$ contains $(-2)$-curves, the above conclusion still holds. Indeed, each connected component of the union of the $(-2)$-curves in $X$ is a Hirzebruch-Jung string. The space $\check{X}$ obtained by blowing down these curves therefore [3] only has A-D-E singularities, and so may be given the structure of a complex orbifold with singularities modeled on $\mathbb{C}^{2} / \Gamma$, $\Gamma \subset S U(2)$. Because $\check{X}$ admits pluri-canonical embeddings into projective space, it has $c_{1}<0$ in the orbifold sense, and the Aubin-Yau proof, without essential alterations, shows $[10,29]$ that it therefore admits an orbifold Kähler-Einstein metric $\check{g}$. Since the Ricci form $\rho$ of $\check{g}$, pulled back to $X$, represents $2 \pi c_{1}$, it follows that

$$
\int_{\check{X}} s_{\check{g}}^{2} d \mu_{\check{g}}=8 \int_{X} \rho^{2}=8\left(2 \pi c_{1}(X)\right)^{2}=32 \pi^{2} c_{1}^{2}(X) .
$$

Choose $\check{g}$-geodesic coordinates around each orbifold point of $\check{X}$, as well as geodesic coordinates around $k$ distinct nonsingular points as before. On the A-D-E resolutions of $\mathbb{C}^{2} / \Gamma$, one can find asymptotically flat hyperKähler metrics [11], and we may modify our previous definition of $g_{t}$ by defining $h_{2}(t)$ on the orbifold charts so that $\delta+t^{4} h_{2}(t)$, rather than being a Burns metric, is instead a Kronheimer metric for which the HirzebruchJung string has area $\propto t^{4}$. The previous estimates are then still valid, and we once again have

$$
\lim _{t \rightarrow 0^{+}} \int_{M} s_{g_{t}}^{2} d \mu_{g_{t}}=\int_{\check{X}} s_{\check{g}}^{2} d \mu_{\check{g}}=32 \pi^{2} c_{1}^{2}(X)
$$

This proves 
Theorem 6. Let $X$ be a minimal algebraic surface of general type, and let $M=X \# k \overline{\mathbb{C P}}_{2}$. Let $\mathcal{M}$ denote the space of Riemannian metrics on M. Then

$$
\inf _{g \in \mathcal{M}} \int_{M} s_{g}^{2} d \mu_{g}=32 \pi^{2}(2 \chi+3 \tau+k),
$$

where $\chi$ and $\tau$ are the Euler characteristic and signature of $M$. Moreover, this infimum is never attained if $k>0$.

The scale-invariant Riemannian functional $\int s^{2} d \mu$ has a less distinguished pedigree than the Hilbert action $\int s d \mu$, which [4] has historically played a central rôle in the theory of Einstein spaces. Because the Hilbert action is not scale invariant, it is usual to restrict it to the set of metrics of volume 1 ; this is equivalent to instead studying the scale-invariant functional

$$
\mathcal{S}(g)=\frac{\int s d \mu}{\sqrt{\int d \mu}} .
$$

Let $\mathcal{C}$ denote the set of conformal classes on $M^{4}$. For each conformal class, one defines the Yamabe constant $Y([g])$ by

$$
Y([g])=\inf _{g \in[g]} \mathcal{S}(g),
$$

and a remarkable result of Yamabe, Trudinger, Aubin, and Schoen [17, 20] asserts that this infimum is always attained by a metric of constant scalar curvature. One may then define an invariant of $M$, known as the Yamabe invariant or sigma constant, by

$$
Y(M)=\sup _{[g] \in \mathcal{C}} Y([g])=\sup _{[g] \in \mathcal{C}} \inf _{g \in[g]} \mathcal{S}(g),
$$

and if this sup inf is attained, any metric which attains it is necessarily Einstein. The following observation shows that we have already essentially calculated the Yamabe invariants of surfaces of general type; cf. [1, Theorem 7.3].

Lemma 1. Let $M$ be a smooth compact 4-manifold, and let $[g]$ be a conformal class with negative Yamabe constant. Then

$$
\inf _{g \in[g]} \int s_{g}^{2} d \mu_{g}=|Y([g])|^{2}
$$

and this infimum is precisely achieved by the metrics of constant scalar curvature. 
Proof. Let $g \in[g]$ be any metric in the conformal class, and let $\hat{g}=\phi^{2} g$ be a metric of constant scalar curvature. The smooth positive function $\phi$ then satisfies

$$
\hat{s} \phi^{3}=6 \Delta \phi+s \phi,
$$

where the scalar curvature $\hat{s}<0$ of $\hat{g}$ is constant. Since the volume form of $\hat{g}$ is $\hat{d \mu}=\phi^{4} d \mu$, we have

$$
\begin{aligned}
\int s^{2} d \mu & =\int\left(\hat{s} \phi^{2}-6 \frac{\Delta \phi}{\phi}\right)^{2} d \mu \\
& =\int \hat{s}^{2} \phi^{4} d \mu-12 \hat{s} \int \phi \Delta \phi d \mu+\int\left(6 \frac{\Delta \phi}{\phi}\right)^{2} d \mu \\
& =\int \hat{s}^{2} \hat{d \mu}+12|\hat{s}| \int|\nabla \phi|^{2} d \mu+\int\left(6 \frac{\Delta \phi}{\phi}\right)^{2} d \mu \\
& \geq \int \hat{s}^{2} \hat{d \mu}=Y([g])^{2} .
\end{aligned}
$$

Moreover, equality only occurs if $\phi$ is constant.

Now if $X$ is a minimal surface of general type, and if $M=X \# k \overline{\mathbb{C} P}_{2}$, some Seiberg-Witten invariant is non-zero for every metric on $M$, and hence the Yamabe constant of every conformal class is negative. Thus

$$
Y(M)=-\inf _{[g] \in \mathcal{C}}|Y([g])|=-\inf _{g \in \mathcal{M}}\left[\int_{M} s^{2} d \mu\right]^{1 / 2}=-4 \pi \sqrt{2 c_{1}^{2}(X)} .
$$

Moreover, the Yamabe minimax is unattained if $k>0$. To summarize:

Theorem 7. Let $M$ be obtained from a minimal surface $X$ of general type by blowing up $k \geq 0$ points. Then the Yamabe invariant $Y(M)$ is independent of $k$, and is given by

$$
Y(M)=-4 \pi \sqrt{2 c_{1}^{2}(X)}
$$

Moreover, this sup inf is unachieved if $k>0$.

It is therefore impossible to construct Einstein metrics on a non-minimal surface of general type by the Yamabe minimax program of $[9,20]$.

Which compact complex surfaces $(M, J)$ actually admit Einstein metrics? Given such a metric $g$, the Hitchin-Thorpe inequality forces $c_{1}^{2}(M)>$ 0 unless $(M, g)$ has a finite isometric cover $(\tilde{M}, \tilde{g})$ which is hyper-Kähler. In the latter case, we may equip $\tilde{M}$ with the pulled-back complex structure $\tilde{J}$, and observe that $(\tilde{M}, \tilde{J})$ is of Kähler type because $b_{1}(\tilde{M})$ is even $[21,3]$; by averaging a Kähler form over the action of the fundamental group, we thus conclude that $(M, J)$ is itself of Kähler type. Moreover, $b^{+}(\tilde{M})=3$, so the Seiberg-Witten invariant of $(\tilde{M},[\tilde{J}])$ is well-defined and 
non-zero. But since $\tilde{M}$ admits a scalar-flat metric, Theorem 1 implies that $c_{1}(\tilde{M}, \tilde{J})$ is zero in real cohomology, and hence that $c_{1}(M, J)$ is a torsion class. Thus $(M, J)$ is a minimal surface of Kodaira dimension 0, and [31] admits a compatible Ricci-flat Kähler metric.

If instead $c_{1}^{2}(M)>0$, the information available is only slightly weaker, as the Kodaira classification of complex surfaces [3] tells us that $M$ is either rational or of general type; in particular, $(M, J)$ is again of Kähler type. On the other hand, Theorem 3 and the above discussion make it seem plausible that non-minimal surfaces of general type never admit Einstein metrics. This leads to the following speculation:

Conjecture 1. Let $(M, J)$ be an irrational compact complex surface. Then $M$ admits an Einstein metric iff it admits a Kähler-Einstein metric compatible with some small deformation of $J$.

The uniqueness theorem of [14] might even lead one to hope for more:

Conjecture 2. Let $(M, J)$ be an irrational compact complex surface. Then any Einstein metric on $M$ is Kähler with respect to some deformation of $J$.

However, further progress on either of these conjectures would seem to require new estimates for $\left|W_{+}\right|^{2}$.

Notice we have excluded the rational surfaces from the above conjectures. The Seiberg-Witten invariants vanish for such surfaces; even worse, $\mathbb{C} P_{2} \# \overline{\mathbb{C P}}_{2}$ admits a non-Kähler Einstein metric, discovered by Page [19]. However, the Page example is conformally Kähler [4], and so does not rule out

Conjecture 3. Let $(M, J)$ be a compact complex surface. Then $M$ admits an Einstein metric iff it admits an Einstein metric which is conformally Kähler with respect to some small deformation of $J$.

Indeed, in light of the highly developed existence theory [22, 27, 28] available for Kähler-Einstein metrics on rational surfaces, this differs from Conjecture 1 only by hypothesizing the existence of an Einstein conformally Kähler metric on $\mathbb{C} P_{2} \# 2 \overline{\mathbb{C} P}_{2}$. For further discussion, see [16].

Finally, let us discuss Gromov's notion of minimal volume [7] and some generalizations thereof. Fix a smooth manifold, and let $\mathcal{M}_{|K|}$ be the set of metrics on $M$ whose sectional curvature $K$ satisfies $|K| \leq 1$. Then Gromov's minimal volume invariant is

$$
\operatorname{Vol}_{|K|}(M):=\inf _{g \in \mathcal{M}|K|} \int_{M} d \mu_{g}
$$


Let us now assume that $M$ is 4-dimensional, and let $\mathcal{M}_{K}, \mathcal{M}_{r}$, and $\mathcal{M}_{s}$ respectively denote the set of metrics satisfying $K \geq-1, r \geq-3 g$ and $s \geq-12$. Then, in the spirit of [5], we may also define invariants

$$
\begin{aligned}
\operatorname{Vol}_{K}(M) & :=\inf _{g \in \mathcal{M}_{K}} \int_{M} d \mu_{g} \\
\operatorname{Vol}_{r}(M) & :=\inf _{g \in \mathcal{M}_{r}} \int_{M} d \mu_{g} \\
\operatorname{Vol}_{s}(M) & :=\inf _{g \in \mathcal{M}_{s}} \int_{M} d \mu_{g}
\end{aligned}
$$

which we shall call the sectional, Ricci and scalar minimal volumes of $M$. Notice that our conventions are chosen so that $\mathcal{M}_{|K|} \subset \mathcal{M}_{K} \subset \mathcal{M}_{r} \subset \mathcal{M}_{s}$; consequently, we automatically have

$$
\operatorname{Vol}_{|K|}(M) \geq \operatorname{Vol}_{K}(M) \geq \operatorname{Vol}_{r}(M) \geq \operatorname{Vol}_{s}(M) .
$$

Now if $M$ is a complex surface of Kodaira dimension $\geq 1$, some SeibergWitten invariant is non-zero for every metric, and for each metric the minimum of the scalar curvature is therefore necessarily negative. Thus $\mathrm{Vol}_{s}$ is the infimum of the volumes of metrics with $\min s=-12$. By rescaling, it follows that

$$
\operatorname{Vol}_{s}(M)=\inf _{g \in \mathcal{M}} \frac{\left(\min s_{g}\right)^{2}}{144} \int d \mu_{g}
$$

The following observation now comes to our aid:

Lemma 2. For a conformal class $[g]$ with negative Yamabe constant on any smooth compact 4-manifold,

$$
\inf _{g \in[g]}\left(\min s_{g}\right)^{2} \int d \mu_{g}=\inf _{g \in[g]} \int s_{g}^{2} d \mu_{g}=|Y([g])|^{2}
$$

and is attained precisely by the metrics of constant scalar curvature.

Proof. Let $g$ again be any metric in $[g]$, and let $\hat{g}=\phi^{2} g$ again be a metric of constant scalar curvature $\hat{s}<0$. At the maximum of $\phi$, the equation

$$
\hat{s} \phi^{3}=6 \Delta \phi+s \phi
$$

tells us that $\hat{s} \phi^{3} \geq s \phi$, so that $\hat{s}(\max \phi)^{2} \geq \min s$, and hence

$$
|\hat{s}|(\max \phi)^{2} \leq|\min s| \text {. }
$$

Thus

$$
(\min s)^{2} \int d \mu \geq \int \hat{s}^{2}(\max \phi)^{4} d \mu \geq \int \hat{s}^{2} \phi^{4} d \mu=\int \hat{s}^{2} \hat{d \mu}=|Y([g])|^{2} .
$$


Moreover, equality occurs only if $\phi$ is constant.

If $X$ is now a minimal surface of general type, and if $M=X \# k \overline{\mathbb{C P}}_{2}$, we therefore have

$$
\operatorname{Vol}_{s}(M)=\inf _{[g] \in \mathcal{C}} \inf _{g \in[g]} \frac{\left(\min s_{g}\right)^{2}}{144} \int d \mu=\inf _{g \in \mathcal{M}} \frac{1}{144} \int_{M} s_{g}^{2} d \mu_{g}=\frac{2 \pi^{2}}{9} c_{1}^{2}(X) .
$$

This proves

Theorem 8. Let $M$ be obtained from a minimal surface $X$ of general type by blowing up $k \geq 0$ points. Then the scalar minimal volume of $M$ is independent of $k$, and is given by

$$
\operatorname{Vol}_{s}(M)=\frac{2 \pi^{2}}{9} c_{1}^{2}(X)>0 .
$$

If $k>0$, no metric on $M$ actually realizes this minimal volume.

In particular, this shows that the Gromov minimal volume $\mathrm{Vol}_{|K|}$ is non-zero for any complex algebraic surface of general type. Of course, one expects that $\mathrm{Vol}_{|K|}>\mathrm{Vol}_{r}>\mathrm{Vol}_{s}$ for most such surfaces, but it is also apparent that $\mathrm{Vol}_{r}=\mathrm{Vol}_{s}$ when $M=X$ is minimal and contains no $(-2)$-curves. It would certainly be interesting to know precisely when these tautological inequalities are actually strict.

Finally, it should be mentioned that Gromov introduced [7, 4] a fundamental-group invariant called the simplicial volume in order to estimate his minimal volume $\mathrm{Vol}_{|K|}$, and observed that this provides a method distinct from the Hitchin-Thorpe inequality for showing that certain 4manifolds with large fundamental group cannot admit Einstein metrics. It is for this reason ${ }^{1}$ that the author chose to focus on simply connected manifolds in Theorem A.

\section{Acknowledgement}

The author would like to warmly thank Michael Anderson and Dusa McDuff for their helpful comments.

\footnotetext{
${ }^{1}$ Shortly after the November, 1995 posting of this article as dg-ga e-print 9511015 , independent infinite- $\pi_{1}$ examples were described by Andrea Sambusetti, An Obstruction to the Existence of Einstein Metrics on 4-Manifolds, Preprint 95-179, December 1995, Ecole Normale Supérieur de Lyon. Sambusetti uses the entropy inequalities of BessonCourtois-Gallot [5] to show, for example, that the connected sum of two copies of any real-hyperbolic 4-manifold never admits Einstein metrics, even though such a space satisfies the strict Hitchin-Thorpe inequality.
} 


\section{References}

1. M. T. Anderson, Extrema of Curvature Functionals on the Space of Metrics on 3-Manifolds, preprint, (1995).

2. T. Aubin, Equations du Type Monge-Ampère sur les Variétés Kählériennes Compactes, C. R. Acad. Sci. Paris 283A (1976), 119-121.

3. W. Barth, C. Peters, and A. Van de Ven, Compact Complex Surfaces, SpringerVerlag, 1984.

4. A. Besse, Einstein Manifolds, Springer-Verlag, 1987.

5. G. Besson, G. Courtois, and S. Gallot, Entropies et Rigidités des Espaces Localement Symétriques de Courbure Strictement Négative, Geom. and Func. Anal. 5 (1995), 731-799.

6. R. Friedman and J. Morgan, Algebraic Surfaces and Seiberg-Witten Invariants, preprint, 1995.

7. M. Gromov, Volume and Bounded Cohomology, Publ. IHES 56 (1982), 5-99.

8. N. J. Hitchin, On Compact Four-Dimensional Einstein Manifolds, J. Diff. Geom. 9 (1974), 435-442.

9. O. Kobayashi, Scalar Curvature of a Metric of Unit Volume, Math. Ann. 279 (1987), 253-265.

10. R. Kobayashi, Einstein-Kähler V-Metrics on Open Satake V-Surfaces with Isolated Quotient Singularities, Math. Ann. 272 (1985), 385-398.

11. P. Kronheimer, Instantons Gravitationelles et Singularités de Klein, C.R. Acad. Sci. Paris 303 (1986), 53-55.

12. P. Kronheimer and T. Mrowka, The Genus of Embedded Surfaces in the Complex Projective Plane, Math. Res. Lett. 1 (1994), 797-808.

13. C. LeBrun, Counter-Examples to the Generalized Positive Action Conjecture, Comm. Math. Phys. 118 (1988), 1205-1208.

14. ___ Einstein Metrics and Mostow Rigidity, Math. Res. Lett. 2 (1995), 1-8.

15. —_ Polarized 4-Manifolds, Extremal Kähler Metrics, and Seiberg-Witten Theory, Math. Res. Lett. 2 (1995), 653-662.

16. ___ Einstein Metrics on Complex Surfaces, in Geometry and Physics, J. Andersen, J. Dupont, H. Pedersen, and A. Swann, eds. Marcel Dekker, to appear.

17. J. Lee and T. Parker, The Yamabe Problem, Bull. Am. Math. Soc. 17 (1987), 37-91.

18. D. McDuff, Blow Ups and Symplectic Embeddings in Dimension 4, Topology 30 (1991), 409-421.

19. D. Page, A Compact Rotating Gravitational Instanton, Phys. Lett. 79B (1979), 235-238.

20. R. Schoen, Variational Theory for the Total Scalar Curvature Functional for Riemannian Metrics and Related Topics, Lec. Notes Math. 1365 (1987), 120-154.

21. Y. T. Siu, Every K3 Surface is Kähler, Inv. Math. 73 (1983), 139-150.

22. __ The Existence of Kähler-Einstein Metrics on Manifolds with Positive AntiCanonical Line Bundle and Suitable Finite Symmetry Group, Ann. Math. 127 (1988), 585-627.

23. C. H. Taubes, The Seiberg-Witten Invariants and Symplectic Forms, Math. Res. Lett. 1 (1994), 809-822.

24. _ More Constraints on Symplectic Forms from Seiberg-Witten Invariants, Math. Res. Lett. 2 (1995), 9-14.

25. __ The Seiberg-Witten and Gromov Invariants, Math. Res. Lett. 2 (1995), 221238. 
26. J. A. Thorpe, Some Remarks on the Gauss-Bonnet Formula, J. Math. Mech. 18 (1969), 779-786.

27. G. Tian, On Calabi's Conjecture for Complex Surfaces with Positive First Chern Class, Inv. Math. 101 (1990), 101-172.

28. G. Tian and S.-T. Yau, Kähler-Einstein Metrics on Complex Surfaces with $c_{1}>0$, Comm. Math. Phys. 112 (1987), 175-203.

29. H. Tsuji, Existence and Degeneration of Kähler-Einstein Metrics on Minimal Algebraic Varieties of General Type, Math. Ann. 281 (1988), 123-133.

30. E. Witten, Monopoles and Four-Manifolds, Math. Res. Lett. 1 (1994), 809-822.

31. S.-T. Yau, On the Ricci-Curvature of a Complex Kähler Manifold and the Complex Monge-Ampère Equations, Comment. Pure Appl. Math. 31 (1978), 339-411.

Department of Mathematics, State University of New York, Stony Brook, NY 11794-3651

E-mail address: claude@math.sunysb.edu 